\title{
Metodologías participativas y Facebook en el ámbito universitario
}

\section{Participatory methodologies and Facebook in Higher Education}

\author{
Nuria Serrat Antoli ${ }^{1}$ \\ ${ }^{1}$ Universidad de Barcelona, España (nserrat@ub.edu)
}

\section{RESUMEN:}

La conexión entre el EEES, las metodologías participativas y las 2.0 es el núcleo de atención esta innovación. Como tal, pretende ir incorporando alguna de las herramientas 2.0 en el marco de la educación superior, con el objetivo de potenciar, reflexionar y aplicar metodologías participativas que el EEES solicita. El proyecto tiene por objetivo general sistematizar, aplicar y valorar un conjunto de metodologías participativas para la adquisición de determinadas competencias transversales y específicas (propias del ámbito de la formación de formadores) y utilizar las 2.0 (en concreto, Facebook) como entorno de intercambio en el que analizarlas y potenciarlas. La innovación ha utilizado dos herramientas básicas de recogida de información: análisis de contenido (el generado por parte de alumnos y docentes en el marco de la asignatura) y encuesta con 10 preguntas cerradas dirigidas al alumnado. Tras la aplicación de la innovación, algunas de las conclusiones pueden resumirse en: la baja participación que se generó en un primer momento; el cuestionamiento sobre la participación de carácter voluntario, que posiblemente se convirtió en un punto débil; destacar la potencialidad de Facebook como generador de conocimiento; el papel decisivo del dinamizador en Facebook; el interés despertado por cuestiones no directamente vinculadas con la asignatura pero sí vinculada con "la vida" del estudiante; y la potencialidad de la plataforma para el intercambio de información relacionada con una determinada temática.

\section{PALABRAS CLAVE: METODOLOGÍA PARTICIPATIVA, DOCENCIA UNIVERSITARIA, FACEBOOK.}

\begin{abstract}
:
The connection between the EHEA, participatory methodologies and 2.0 is the core of attention this innovation. As such, intends to incorporate some of Web 2.0 tools in the context of higher education, with the aim of promoting, reflect and apply participatory methodologies to the EHEA requested. The project's overall objective is to systematize, implement and evaluate a set of participatory methodologies to acquire certain transversal and specific competences (specific to the field of training of trainers) and use the 2.0 (specifically, Facebook) as exchange environment which analyze them and empower them. Innovation has used two basic tools of information collection: content analysis (generated by students and teachers in the context of the assignatura) and survey with 10 closed questions aimed at students. After application of innovation, some conclusions can be summarized as: low turnout that was generated at first; questioning on voluntary participation, possibly became a weak point; highlight the potential of Facebook as a generator of knowledge; the crucial role of facilitator in
\end{abstract}


Facebook; the interest in issues not directly related to the subject but if linked to "life" of the student; and potential of the platform for the exchange of information related to a particular topic.

\section{KEYWORDS: PARTICIPATORY METHODOLOGY, UNIVERSITY TEACHING, FACEBOOK.}

\section{CONTEXTUALIZACIÓN DE LA INNOVACIÓN}

La implantación del proceso de "convergencia europea" pone de manifiesto una serie de retos que afectan a distintas áreas de la docencia universitaria. Uno de ellos es que cambia la orientación del proceso formativo que prevalecía hasta el momento (centrado casi exclusivamente en el docente) hacia una perspectiva en la que el eje vertebrador del proceso de enseñanzaaprendizaje es el alumnado (Font, 2003; Goñi, 2005; Blanco, 2009; Teichler, 2010). Poco a poco, en nuestro sistema educativo van consolidándose nuevas metodologías docentes, nuevas herramientas de evaluación y tutoría, y nuevos materiales didácticos centrados en el modelo del student-centered learning (Niemi, 2009; Light, Cox y Calkins, 2009). Desde el punto de vista del profesorado, se demanda ahora un nuevo perfil docente que pueda fomentar en el alumnado aprendizajes más significativos, habilidades de pensamiento superior, el aprender a aprender mediante la revisión del ejercicio profesional y la habilidad para el desarrollo del pensamiento reflexivo. Todas estas propuestas y el desarrollo y aplicación de las mismas, están exigiendo al profesorado adaptar y transformar su conocimiento y concepciones, y, especialmente, sus hábitos, a una gran diversidad de enfoques y alternativas que, de manera combinada, conduzcan al alumnado hacia los nuevos objetivos de aprendizaje. Bajo este nuevo prisma, la metodología docente se convierte en una de las piedras de toque, requiriendo una revisión que huya de estrategias tradicionales y marcadamente academicistas para incorporar metodologías participativas capaces de situar al alumno en la primera línea del proceso de enseñanza-aprendizaje.

En este mismo contexto de implantación del EEES, se está produciendo en paralelo una progresiva introducción de las herramientas 2.0 en las instituciones universitarias. La formación del estudiante se abre a una multitud de espacios y recursos curriculares sincrónicos y asincrónicos hasta el momento apenas contemplados en las prácticas de la enseñanza universitaria. Varios estudios confirman que se ha producido en los últimos 7 años un aumento más que significativo del uso de estos recursos en la educación superior, y su empleo por parte de docentes y estudiantes va en aumento (aunque no sin despertar ciertas reticencias a nivel institucional, Serrat y Rubio, 2012). Su aplicación ha sido estudiada de manera incipiente desde distintos puntos de vista: como herramienta de socialización y pertenencia al grupo (Madge y al., 2009; Llorens y Capdeferro, 2011); como espacio de comunicación entre docentes y alumnos (Cheung y Vogel, 2011); como espacio de generación colaborativa de conocimiento (Wheeler, 2009; Buchem y Hamelmann, 2011); como herramienta que potencia y enriquece la construcción de capital social (Valenzuela y al., 2009) o como entorno de aprendizaje colaborativo y aprendizaje informal (Serrat y Rubio, 2011).

Como cualquier otra nueva incorporación en el ámbito educativo, las voces críticas o fervientes emergen en multitud de foros y plataformas de discusión, utilizando tanto los tradicionales canales de comunicación como las propias herramientas 2.0 con las que comulgan. En este sentido, muchos son los focos de discusión y las perspectivas de futuro inmediato.

En todo caso, el punto de conexión entre el EEES, las metodologías participativas y las 2.0 fue el núcleo de atención de la innovación que aquí se describe. Como tal, pretendía ir incorporando alguna de las herramientas 2.0 en el marco de la educación superior, con el objetivo de potenciar, reflexionar y aplicar algunas de las metodologías participativas que el EEES solicita. Como argumentan Espuny y otros (2011:173), "las redes sociales, por encima de cualquier otra consideración, tienen mucho que ver con las nuevas metodologías participativas que de modo masivo se están adoptando en el espacio europeo de educación superior". Es en este sentido que una nueva mirada se aplicó a la asignatura troncal de la Facultad de Pedagogía de la Universidad de Barcelona "Diseño, desarrollo y evaluación de la formación" ( $6^{\circ}$ semestre del Grado de Pedagogía), orientada, entre otros aspectos, a que el alumnado aplicase, consolidase y transfiriese metodologías participativas (Serrat y Jarauta, 2011) que pudieran ser de utilidad en su futuro profesional, ya sea 
como formadores o como gestores de la formación.

\section{DISEÑO DE LA INNOVACIÓN Y METODOLOGÍA}

El proyecto1 "Metodología participativa, docencia universitaria y herramientas 2.0 " tenía por objetivo general sistematizar, aplicar y valorar un conjunto de metodologías participativas para la adquisición de determinadas competencias transversales y específicas (propias de la formación) y utilizar las 2.0 (en concreto, Facebook) como entorno de intercambio en el que analizarlas y potenciarlas. La introducción de estas metodologías participativas tenía un significado y un peso específicos en nuestra asignatura por cuanto los estudiantes que las recibían como objeto de estudio $\mathrm{y}$ en las que participaban como parte de su aprendizaje, debían aplicarlas en su futuro inmediato como profesionales de la Pedagogía, y en concreto, de la formación. Es decir, las metodologías participativas en este caso no sólo formaban parte de su proceso cotidiano de aprendizaje en el aula, sino que constituían parte del contenido que debían conocer y aplicar en su práctica profesional futura.

La metodología forma parte intrínseca del contenido de aprendizaje.

Además de la adquisición y desarrollo de las mismas, el equipo docente incorporó un perfil de Facebook mediante el cual se reflexionaba acerca de la aplicación de dicha metodología, complementando con otros contenidos aquello trabajado en el aula. Como argumentan Llorens y Capdeferro (2011:33), aunque "Facebook es un ejemplo de red social 2.0 que presenta un gran potencial en la educación, a pesar de no haber sido concebida como un entorno para construir y gestionar experiencias de aprendizaje", consideramos que la posibilidad de poder construir y ampliar nuevo conocimiento a través de Facebook era una buena oportunidad para una

\footnotetext{
${ }^{1}$ La innovación "Formación 2.0: Metodologías participativas y redes sociales en el ámbito universitario", dirigida por Dra. Núria Serrat, ha sido posible gracias al Programa de Innovación Docente del Programa de Mejora e Innovación Docente de la Universidad de Barcelona 2011. Forma parte del conjunto de actuaciones del Grupo de Investigación consolidado "FODIP" (Formación Docente e Innovación Pedagógica, 2009 SGR246) y Grupo de Innovación Consolidado de la Universidad de Barcelona. Se inició en el curso 2011-2012, en la asignatura obligatoria del Grado de Pedagogía de dicha universidad, primer año de impartición tras la consolidación del EEES en la Universidad de Barcelona
}

asignatura que pretende formar a futuros formadores.

\subsection{Objetivos de la innovación}

Teniendo en cuenta este background, los objetivos de la innovación fueron:

- Aplicar de manera sistemática metodologías participativas que constituyan a la vez método y contenido en el marco de la asignatura "Diseño, desarrollo y evaluación de la formación". Se trata de consolidar los aprendizajes fundamentales de la asignatura a través de la aplicación de estrategias que, posteriormente, a los estudiantes les sean de utilidad en su ámbito profesional.

- Validar qué competencias básicas y específicas (vinculadas al mundo de la formación) se incorporan a partir de la utilización de las metodologías participativas aplicadas en la asignatura, y cómo las 2.0 pueden colaborar a desarrollarlas o potenciarlas.

- Incorporar Facebook como herramienta de intercambio, reflexión, ampliación y profundización de las diferentes metodologías participativas aplicadas. Aunque no se ha concebido como elemento central de la asignatura, la utilización de FB se ha incorporado con el objetivo de compartir y consolidar determinadas competencias y conocimientos relativos al ámbito de la formación.

\subsection{Muestra y condiciones de aplicación}

La innovación se aplicó durante el curso 20112012 a un total de 190 alumnos, los matriculados a los grupos M1, M2, T1 y T2 de tercero del Grado de Pedagogía. Todos ellos eran alumnos de la asignatura de "Diseño, Desarrollo y Evaluación de la Formación" contexto en el que se puso en práctica la experiencia.

Desde el punto de vista de las condiciones de aplicación de la innovación existen algunas particularidades a comentar que afectaron a tres aspectos clave:

1. El conjunto de metodologías participativas aplicadas en el aula.

2. El papel del alumnado con relación al Facebook de la asignatura.

3. El papel del profesorado con relación al Facebook de la asignatura. 
a) El conjunto de estrategias metodológicas participativas aplicadas en el aula.

Las metodologías participativas elegidas para la innovación en el marco de la asignatura fueron: 4 esquinas, taller dirigido, puzzle de grupos, red de conceptos, colocación de estructuras y estudio de caso, todas ellas utilizadas de forma frecuente en el ámbito de la formación. Estas metodologías implantadas en el aula fueron trabajadas para que el alumnado las pudiese conocer a partir de la participación directa en las mismas. Se trabajaron de manera reflexiva conjuntamente con el profesorado para extraer los aprendizajes derivados de las mismas y fueron valoradas de manera colaborativa vía facebook.

b) El papel del alumnado con relación al Facebook de la asignatura

Desde el punto de vista del alumnado, algunos de los aspectos que se consideraron fueron:

- La participación en el Facebook de la asignatura, como primer año de aplicación, se planteó como voluntaria. El alumnado sabía, desde el inicio de la asignatura, que la herramienta era un complemento al conjunto de la asignatura, y que no formaba parte de la evaluación de la misma. Finalmente, se dieron de alta un total de 142 de 190 alumnos, un 74\% (hasta los 190, algunos no quisieron participar, otros no tenían Facebook).

- Como normas básicas de participación, se estableció que se podía compartir, aportar y debatir sobre cualquier contenido relacionado con la asignatura, aunque también podían aportarse informaciones de interés relacionadas con el mundo de la formación en general. El tipo de contenidos compartidos fueron: links a webs de interés, vídeos, noticias, y comentarios personales.

- Se eliminaba la información personal de los alumnos. Es decir, para filtrar aquella información de carácter personal que el alumnado publicaba en su correspondiente muro, a cada nueva alta el profesorado se encargaba de dar de baja el canal de noticias del alumno. Así pues, el profesorado evitaba entrar en la parcela privada de los alumnos, información nada relevante para el desarrollo de la asignatura y de la innovación. Ello entendemos que también dio cierto margen para que el alumnado se sintiera más cómodo al saber que el profesorado evitaba observar los aspectos publicados de forma individual en cada página personal.

c) El papel del profesorado con relación al Facebook de la asignatura

Desde el punto de vista del profesorado, algunos de los aspectos que se consideraron fueron:

- Se creó un perfil único para el conjunto de la asignatura, de manera que no aparecían los nombres de cada uno de los miembros del profesorado. Ello permitía actuar bajo un perfil único, a la vez que no obligaba a los docentes a darse de alta con sus perfiles privados. Ello permitía también que todo el profesorado pudiera gestionar el perfil según sus necesidades, atendiendo a temáticas de interés que hubieran podido aparecer en el aula.

- Los roles adoptados por parte del profesorado se materializaron en:

- Motivador de nuevas temáticas.

- Provocador.

- Facilitador de información.

Como aspecto general, se consensuó que el profesorado no adoptaría un rol fiscalizador, censor o controlador, facilitando así la incorporación de opiniones abiertas por parte del alumnado.

- Para el análisis y reflexión de los contenidos trabajados en el aula, y en especial, de las metodologías participativas utilizadas en el aula, el profesorado fue planteando 4 tipologías básicas de preguntas:

- Preguntas introductorias o de motivación. Se trata de cuestiones planteadas al inicio de cada nuevo tema, y pretendían motivar hacia los contenidos que se desarrollarían a lo largo de un módulo. Este tipo de preguntas se materializaron básicamente, mediante cuestionarios de respuesta múltiple o preguntas abiertas vinculadas a un vídeo o link.

- Preguntas de consolidación de contenidos. Generalmente planteadas después de haber tratado un determinado contenido o aplicado en el aula una de las metodologías participativas seleccionadas, intentaban profundizar sobre varios aspectos de las mismas.

- Preguntas de transferencia. También planteadas generalmente después del aula, las preguntas de transferencia apelaban a las posibilidades de aplicación de los contenidos tratados a su futuro ámbito laboral (vinculado a la formación). 
- Preguntas de reflexión / opinión. Para reflexionar sobre algunos de los aspectos relativos a la asignatura, o bien a temas vinculados con el mundo de la formación y la educación.

- Se fueron añadiendo otro tipo de informaciones que, bajo criterio del profesorado, podían ser de interés para el alumnado, y siempre relacionadas con la asignatura. Por ejemplo, novedades editoriales con pequeños comentarios, páginas informativas de colegios profesionales y honorarios relativos a la formación, etc. No se utilizó esta herramienta, en cambio, para informar de aspectos organizativos o logísticos de la asignatura, ya que ello se hacía a través del aula virtual específica en la que todos los alumnos (los 190) estaban dados de alta.

\section{METODOLOGÍA}

Desde un punto de vista metodológico, la innovación se desarrolló según los siguientes pasos:

En primer lugar, se realizó un estudio y selección de metodologías participativas centradas en el aprendizaje del alumnado. En el seno del equipo docente, y de manera previa al diseño e implementación de la experiencia, se llevó a cabo un estudio y discusión en torno a aquellas estrategias que más se adecuaban a distintos aspectos: los objetivos que el equipo docente deseaba alcanzar, los contenidos de la asignatura, el tamaño de los grupos, la interacción que se pretendía crear, el tiempo disponible, las características de los alumnos y las diferentes habilidades y estilos docentes existentes. De esta primera fase de análisis y reflexión, surgieron un número importante de estrategias dirigidas a fomentar la participación del alumnado en el aula y su autonomía en el proceso de aprendizaje. Como hemos comentado, las finalmente seleccionadas para su análisis ${ }^{2}$ en el aula fueron: 4 esquinas, taller dirigido, puzzle de grupos, red de conceptos, colocación de estructuras y estudio de caso.

Cabe señalar que una de las características definitorias de la asignatura era la formación "pedagógica" de los que después formarán a otros. En la asignatura, se formaba sobre la formación y

\footnotetext{
${ }^{2}$ En el aula se aplican otras metodologías participativas, que en este caso no han sido núcleo de análisis por parte de este estudio
}

se enseñaba a los que tendrán que enseñar. De este modo, el método que utilizaba el profesorado era el objeto sobre el que versa el contenido de la asignatura, por lo que tan importante resulta aquello que se verbalizaba en las clases como aquello que sucedía y, especialmente, cómo sucedía. La asignatura no pretendía, pues, transmitir de forma mecánica un conjunto de técnicas o estrategias, sino que pretendía implicar a los alumnos en un proceso participativo y reflexivo en el cual el método puesto en marcha en el aula formase parte inseparable del contenido.

Para poder aplicar, de manera adecuada, las estrategias participativas, se articularon un conjunto de acciones de formación interna dirigidas al profesorado de la asignatura, considerando en todo momento la diversidad existente en cuanto a su experiencia docente. En este sentido, el perfil del equipo implicado en la innovación fue muy variado: un catedrático, un profesor titular, dos profesoras contratadas doctoras, una profesora asociada y una profesora postdoctoral. Cabe decir que algunos de los docentes no habían puesto en práctica parte de las estrategias seleccionadas, de modo que fue necesario realizar diversas sesiones de formación interna. En estas sesiones se desarrollaron interesantes procesos de reflexión sobre la propia práctica y la reconstrucción colaborativa de la asignatura atendiendo a las nuevas estrategias de enseñanza que se deseaban introducir.

Estas sesiones de formación adoptaron la modalidad de seminarios internos y la mayoría de ellos fueron impartidos por los/as profesores más experimentados/as en el uso de metodologías participativas. Además de estos seminarios, se recurrió a la observación (no participante y participante) como estrategia de formación. De este modo, parte del equipo pudo aprender cómo docentes más experimentados utilizaban las nuevas estrategias en el aula. Y los docentes más experimentados, observaron y analizaron como otros profesores con menos experiencia aplicaban las estrategias.

La tercera fase consistió, propiamente, en la aplicación de las metodologías participativas en el aula. Todas las sesiones tuvieron una duración de 2 horas, y todas las metodologías utilizaron este tiempo (excepto el taller dirigido y el estudio de caso, con 4 horas cada una). La mayoría de estrategias fueron implementadas por dos formadores, manteniendo (por razones de 
distribución horaria) las parejas de M1 y T1, y M2 y T2.

Apertura, dinamización y seguimiento de la página de Facebook, desde el inicio de la asignatura. Las informaciones y preguntas se generaron a partir de los contenidos trabajados en el aula, y las aportaciones realizadas por parte del alumnado.

Precisamente, para estas dos últimas fases, se idearon dos instrumentos de recogida de información que nos permitieron una vez implantada la innovación, conocer hasta qué punto se habían conseguido los objetivos fijados. Los instrumentos fueron:

- Análisis de contenido generado en Facebook. Especialmente aplicando una categorización inductiva (a partir de los datos obtenidos) y por repetición.

- Encuesta con 10 preguntas cerradas que se distribuyó a todo el alumnado que participó en la experiencia. $\mathrm{Su}$ objetivo era validar la idoneidad de las metodologías implantadas y evidenciar las competencias básicas y específicas adquiridas.

\section{IMPLANTACIÓN DE LA INNOVACIÓN}

Esta innovación se inició en febrero del 2012, coincidiendo con el inicio de la asignatura en el Grado de Pedagogía en el segundo semestre de tercer curso. Todas las estrategias metodológicas participativas ideadas pudieron ser implantadas en los cuatro grupos.

Teniendo en cuenta que Facebook aportó el primer gran bloque de contenido, fue el foco de atención de la primera recogida y análisis de la información, vaciando desde el inicio de la asignatura un conjunto de unidades de significado.

\section{APORTACIONES DEL ALUMNADO}

Desde el punto de vista de la participación general, podríamos decir que ésta se mantenía entre el 8 y el $12 \%$ del conjunto del alumnado, ciertamente muy baja a la inicialmente esperada. Esta participación se materializó en:

- "Me gusta", tanto a partir de aportaciones de alumnos como del profesorado (aplicado a links, vídeos, lecturas, etc.): 133

- Comentarios del alumnado, a partir de aportaciones realizadas por parte del profesorado y del alumnado: 40 .
- Respuestas a preguntas cerradas: 17.

- Apertura de nuevas líneas de debate por parte de los alumnos: 26.

Respecto a las aportaciones realizadas por parte del alumnado, éstas fueron:

- Vídeos ilustrativos de contenidos trabajados en el aula.

- Links con información complementaria.

- Noticias y comentarios relativos a contenidos de la asignatura.

- Comentarios respecto a aportaciones hechas por parte de los compañeros.

- Comentarios respecto a aportaciones hechas por parte de los docentes.

\section{SOBRE LAS DISTINTAS PREGUNTAS PLANTEADAS}

Uno de los núcleos de interés era las respuestas que el alumnado hacía a las distintas preguntas planteadas. Respecto a las preguntas de motivación inicial, hasta la finalización del curso se aportaron hasta 3 preguntas (una por cada nuevo módulo, excepto los módulos 1 y 3). Éstas han tenido un resultado desigual en cuanto a participación del alumnado:

- Una se realizó en base a la visualización de un vídeo para su posterior comentario. Obtuvo solo 3 comentarios, aunque muy pertinentes al contenido que posteriormente se iba a desarrollar.

Los comentarios se centraron en parte sustantiva del contenido que posteriormente se trabajó en el aula. Por ejemplo:

Se presenta una gran escasez de comunicación verbal, es decir, el lenguaje oral y, en cambio, una abundante comunicación no verbal. La expresión corporal es clave para que el receptor nos pueda entender bien y captar que queremos transmitir. La mayoría de nuestros gestos los adquirimos y exteriorizamos de forma inconsciente y es por ello que el lenguaje corporal siempre refleja lo que realmente sentimos, y no como cuando comunicamos con palabras, ya que a veces podemos mentir. (FB6-M2) (Comentario realizado a partir del visionado de un vídeo sobre la comunicación verbal y no verbal).

(...) La comunicación no verbal refuerza el mensaje que transmite el formador/a, dándole énfasis y sentido. Además, ayuda a que llegue más fácilmente a los alumnos/usuarios, a captar su atención y a ser más coherente. Por tanto, es totalmente necesaria la comunicación verbal y no verbal para desarrollar 
una buena práctica educativa de un buen formador/a. (FB6-M2) (Comentario realizado a partir del visionado de un vídeo sobre la comunicación verbal y no verbal).

- La segunda, tenía que ver con el aprendizaje de determinadas metodologías participativas aplicadas a la formación. Se materializó a partir de una pregunta cerrada de respuesta múltiple, y obtuvo 43 votaciones.

Con relación a las preguntas de consolidación de contenidos, se fueron planteando a lo largo del semestre. Realizadas siempre después del tratamiento en el aula de un determinado contenido, se procuró enfatizar en aspectos clave que pudieran ayudar al alumnado a afianzar un determinado concepto, idea, procedimiento, etc.

Con relación a las preguntas de transferencia, orientadas a poder visualizar aplicaciones posibles de los contenidos trabajados en el aula a contextos profesionales presentes o futuros, se focalizaron, también, en las metodologías participativas, así como otras estrategias puestas en marcha en el aula.

Por último, preguntas de opinión o reflexión general, acerca de contenidos que, si bien no eran núcleo central de la asignatura, sí despertaron un gran interés de los estudiantes, teniendo la educación como punto común de discusión. En especial, queremos hacer mención de un acontecimiento sucedido en el aula, y que despertó ciertos comentarios: algunos alumnos firmaron una determinada actividad en nombre de otros, y esto se transfirió al espacio virtual por parte del profesorado para su comentario.

\section{RELEVANCIA DE LA INNOVACIÓN Y CONCLUSIONES FINALES}

La relevancia del proyecto de innovación entendemos que radica en distintos puntos:

a) En cuanto al alumnado que ha participado en el proyecto:

- Participación directa en un conjunto de metodologías participativas que se han convertido en espacio de desarrollo de competencias propias de la formación, siendo a la vez contenido y método de aprendizaje. El alumnado ha conocido de primera mano en qué consisten y cómo se aplican estas metodologías, a la vez que se ha reflexionado conjuntamente con el alumnado respecto a su potencialidad pedagógica.
- Adquisición y desarrollo de un conjunto de competencias básicas y específicas vinculadas al mundo de la formación.

- Visualización de les posibilidades de aplicación de las metodologías participativas en los futuros puestos de trabajo como pedagogos.

- Utilizar las 2.0 como plataforma de aprovechamiento y consolidación de contenidos.

b) En cuanto al profesorado de la asignatura:

- Adquisición, consolidación y aplicación de metodologías participativas en acorde con los parámetros del EEES.

- Conocimiento más profundo de metodologías participativas que pueden colaborar a generar un aprendizaje centrado en el alumnado.

- Trabajo en equipo y coordinación interna relativa a la puesta en práctica de las metodologías participativas.

- Aprendizaje y reflexión sobre la propia práctica docente mediante la participación en un proceso de innovación que requiere de la creación de espacios colaborativos de reflexión y de búsqueda de nuevas y mejores formas de enseñanza universitaria.

- Conocer las potencialidades de las 2.0 para mejorar la docencia universitaria.

Algunas de las conclusiones extraídas de la aplicación de la innovación, focalizando en la utilización del Facebook en el marco de la asignatura son:

1. En primer lugar, destacar la baja participación que se generó en un primer momento. Nos movíamos en un tanto por ciento muy bajo. No obstante, pudimos analizar sus motivos a partir de los cuestionarios compartidos con los alumnos. Los tres que obtuvieron más respuestas fueron: falta de tiempo; uso no formativo de Facebook; baja motivación respecto a los temas de discusión.

2. En segundo lugar, comentar que lo que fue una puerta abierta a la participación de carácter voluntario, posiblemente se convirtió en un punto débil. Se supuso que el alumnado iba a participar de forma más generalizada en la construcción de contenido. Entendemos que la participación en este tipo de espacios tiene que ser abierta, y por lo tanto, debemos analizar con mucho detalle varios aspectos como los motivos para participar o las temáticas que han generado más o menos interés. 
3. En tercer lugar, visualizar la potencialidad del Facebook como generador de conocimiento. Entendemos, a tenor de los datos generados en la plataforma, que Facebook permitió visualizar el conocimiento aprendido en el aula $y$, especialmente, transferirlo a situaciones personales y profesionales presentes.

4. También comentar el papel decisivo del dinamizador del Facebook. La figura de una persona (o varias) que fueran capaces de generar flujo de información se convirtió en un aspecto básico, ya fueran los docentes o bien los propios alumnos.

5. A su vez, constatamos el interés despertado por cuestiones no directamente vinculadas con la asignatura, pero que tienen relación con lo que podríamos denominar "la vida" del alumnado. Temas como tasas universitarias, movimientos sociales, etc. han sido foco de atención y participación, generando varios comentarios.

6. Por último, observamos la potencialidad de la plataforma para el intercambio de información relacionada con una determinada temática. A pesar de la baja participación, mucha de la que se produjo tuvo la exposición de nueva información (vía link, vídeos, etc.) como uno de los núcleos de contenido.

Teniendo en cuenta los resultados obtenidos en las conclusiones y considerando los diferentes motivos que han llevado a la baja participación de los usuarios, estamos abiertos a desarrollar y consolidar el uso de Facebook en el aula universitaria. Y más si permite reflexionar sobre aspectos vinculados con los núcleos temáticos de las asignaturas, yendo más allá de lo que sucede en la interacción presencial entre docente y alumno.

\section{REFERENCIAS}

Blanco, A. (coord.) (2009). Desarrollo y evaluación de competencias en Educación Superior. Madrid: Narcea.

Buchem, I., y Hamelmann, H. (2011). Developing 21st century skills: Web 2.0 in Higher Education - A case study. eLearning Papers, 24, 1-5.

Cheung, R., y Vogel, D. (2011). Can Facebook enhance the communication between teachers and students? The International Journal of Learning, 17(11), 1-18.

Espuny, C.; González, J.; Lleixà, M.; y Gisbert, M. (2011). Actitudes y expectativas del uso educativo de las redes sociales en los alumnos universitarios. Revista de Universidad y Sociedad del Conocimiento (RUSC), 8(1), 171-185.

Font, A. (2003). El Sistema de Créditos ECTS. Conferencia pronunciada el día 25 de abril de 2003 en el marco de la IX Conferencia de Decanos de las Facultades de
Derecho de las Universidades españolas. Consultado en [http://www.ub.es/mercanti/barcelona2003.pdf], Enero de 2009.

Goñi, J. M. (2005). El espacio europeo de educación superior, un reto para la Universidad. Competencias, tareas y evaluación, los ejes del curriculum universitario. Barcelona: Octaedro/ICE-UB.

Light, G., Cox, R., y Calkins, S. (2009). Learning and teaching in Higher Education. The Reflective Professional. London: Sage. Second Edition.

Madge, C., Meek, J., Wellens, J., y Hooley, T. (2009). Facebook, social integration and informal learning at university: "It is more for socialising and talking to friends about work than for actually doing work", Learning, Media and Technology, 34(2), 141-155.

Niemi, H. (2009). Why from teaching to learning? European Educational Research Journal, 8(1).

Llorens, F.; y Capdeferro, N. (2011). Posibilidades de la plataforma Facebook para el aprendizaje colaborativo en línea. Revista de Universidad y Sociedad del Conocimiento (RUSC), 8(2), 31-45.

Serrat, N., y Jarauta, B. (2011). Active and collaborative methodologies to help improve the learning of future pedagogues. Journal of Teaching and Learning in Higher Education, 3(1), 67-69.

Serrat, N., y Rubio, A. (2012). Coming from outside the Academy. Values and 2.0 culture in higher education. Interactive Learning environments, iFirst.

Teichler, U. (2010). Sistemas comparados de educación superior en Europa. Marcos conceptuales, resultados empíricos y perspectivas de futuro. Barcelona: Octaedro/ICE-UB.

Valenzuela, S., Park, N., Kerk, F. K. (2009). Is There Social Capital in a Social Network Site?: Facebook Use and College Students' Life Satisfaction, Trust, and Participation. Journal of Computer-Mediated Communication, 14, 875-901.

Wheeler, S. (2009). Learning Space Mashups: Combining Web 2.0 Tools to Create Collaborative and Reflective Learning Spaces. Future Internet, 1, 3-13, Retrieved April 22, 2011 from http://www.mdpi.com/19995903/1/1/3/pdf. 